\title{
A Separation and Decoding algorithm for QPSK Signals with Low Complexity
}

\author{
Baijun Pan ${ }^{1, a}$, Shilong Tu² \\ ${ }^{1}$ Science and Technology on Blind Signal Processing Laboratory, Chengdu, china \\ ${ }^{2}$ Science and Technology on Blind Signal Processing Laboratory, Chengdu, china
}

\begin{abstract}
In this paper, we consider separation and decoding for two QPSK signals, which have the same symbol rates. However, the complexity of the present method is too high. To solve this question, an algorithm based on lattice reduction and joint iteration is proposed to enhance the separation performance. The complexity is greatly reduced while retains considerable performance. Simulation results are provided to illustrate the performance of the proposed algorithm.
\end{abstract}

\section{Introduction}

PCMA is a new type of satellite communication multiple access technology[1], which can not only increase the frequency utilization ratio of satellite channel, but also improve the anti-interception capability of communication. So, during the past decades, it has become the research focus of signal processing. In the communication of PCMA, the third party obtains the mixed signal of the same frequency band, and the target signal can not be demodulated by the traditional method. There are two effective methods in the separation of PCMA signal at present. One is the separation of timefrequency mixing signals based on particle filter, which has good separation performance and good error performance, but in order to guarantee the performance of the algorithm, it requires increasing the number of particles, so the computational complexity is large, and the complexity increases exponentially with the modulation order of the signal. The second is the PSP algorithm, which is also of high complexity and is not suitable for processing high order modulation signals. In recent years, the development of lattice reduction techniques in the field of wireless communication is more and more widely used. Lattice reduction technology is originally used in MIMO detection[2]-[5], which enhance the symbol detection performance while reduce the complexity of the algorithm.

In this paper, we propose a low complexity iterative separation and decoding algorithm based on lattice reduction[6]. The remaining part of this paper is organized as follows. The reception model of coded PCMA signals is given in the next section. The section 3 describes the low complexity PCMA joint iterative separation and decoding algorithm. The simulation results are provided in Section 4. Finally, conclusions are drew in Section 5.

\section{Basic signal model}

PCMA is the superposition of two identical frequency modulated signals. The signal model at encoding is shown in Fig. 1

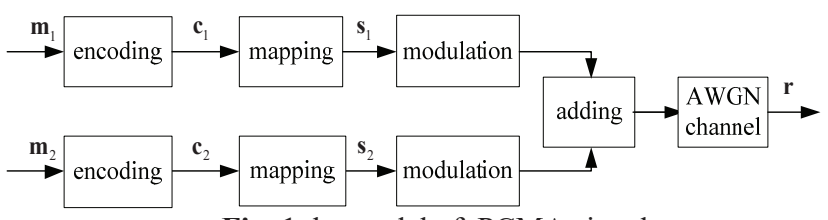

Fig. 1 the model of PCMA signal

The received signal may be expressed as $r(t)=h_{1} e^{j\left(\Delta \omega_{1} t+\theta_{1}\right)} x_{1}(t)+h_{2} e^{j\left(\Delta \omega_{2} t+\theta_{2}\right)} x_{2}(t)+v(t)(1) \quad$ where $h_{1}$ and $h_{2}$ are amplitude fading of two signals respectively, $\Delta \omega_{1}$ and $\Delta \omega_{2}$ are carrier offsets of two signals, $\theta_{1}$ and $\theta_{2}$ are the carrier phase of two signals, $v(t)$ is the additive Gauss white noise, with the mean value is zero and the single power spectrum density is $N_{0}$, and the $x_{\mathrm{i}}(t)$ is a baseband digital modulation signal that can be represented as

$$
x_{i}(t)=\sum_{n=-\infty}^{+\infty} s_{i, n} g_{i}\left(t-n T+\tau_{i}(t)\right)(i=1,2)
$$

where $s_{i, n}$ is the transmitted modulation symbol sequence of the $i$ th signal, $g_{i}(t)$ is the pulse responses of the equivalent channel filters which consist of shaping filters, a physical transmission channel, and a receiving filter, $T$ is the symbol period, and $\tau_{i}(t)$ are the relative 
time delays between the two received modulated signals and the local clock reference.

Sampling the received signal at rate $2 / T$, we can write the discrete-time baseband signal as

$$
\begin{aligned}
& r_{2 k+m}=h_{1} e^{j\left(\Delta \omega_{1}(k T+m T / 2)+\theta_{1}\right)} x_{1,2 k+m} \\
& +h_{2} e^{j\left(\Delta \omega_{2}(k T+m T / 2)+\theta_{2}\right)} x_{2,2 k+m}+v_{2 k+m}
\end{aligned}
$$

where $r_{2 k+m}=r(k T+m T / 2), x_{i, 2 k+m}=x_{i}(k T+m T / 2)$, $v_{2 k+m}=v(k T+m T / 2), k=0,1,2, \cdots$ stands for symbolic moments, $m=0,1$ represents the two sampling times at the same symbolic moment.

Assuming that $g_{i}(t)$ affects only a total of $L$ symbols before and after, the expression is

$$
x_{i, 2 k+m}=\sum_{n=1-L_{1}}^{L_{2}} s_{i, k+n} g_{i}\left(-n T+\tau_{i, 2 k+m}\right)
$$

where $\tau_{i, 2 k+m}=\tau_{i}(k T+m T / 2), L_{1}+L_{2}=L$.

Bring the formula(4) into the formula(3), and

$$
f_{i, 2 k+m}(n)=h_{i} e^{j\left(\Delta \omega_{i}(k T+m T / 2)+\theta_{i}\right)} g_{i}\left(-n T+\tau_{i, 2 k+m}\right)
$$

Then we can get

$$
\begin{aligned}
r_{2 k+m} & =\sum_{n=1-L_{1}}^{L_{2}} s_{1, k+n} f_{1,2 k+m}(n) \\
& +\sum_{n=1-L_{1}}^{L_{2}} s_{2, k+n} f_{2,2 k+m}(n)+v_{2 k+m}
\end{aligned}
$$

In the first separation, we use decision feedback equalization(DFE) based on lattice reduction to detect symbol. Firstly, the channel matrix is extended, that is

$$
\underline{\boldsymbol{H}}_{f}=\left(\begin{array}{c}
\boldsymbol{H}_{f} \\
\sigma I_{K(\tau+1)}
\end{array}\right)
$$

where $\boldsymbol{H}_{f}$ is feedback channel matrix, $\sigma^{2}$ is the variance of $v(t)$, and $I_{K(\tau+1)}$ is the identity matrix. $\boldsymbol{G}_{f}=\underline{\boldsymbol{H}}_{f} \boldsymbol{U}$ is the transformed channel matrix, $Y=\boldsymbol{G}_{f} \overline{\boldsymbol{S}}_{f}+\boldsymbol{n}$, then we can get $\overline{\boldsymbol{S}}_{f}(n)=\boldsymbol{U}^{-1} \boldsymbol{S}_{f}(n)$, where $\boldsymbol{U}$ is unimodular[7]. By conducting pseudo inverse on matrix $\boldsymbol{G}_{f}$, then we obtain

$$
\overline{\boldsymbol{S}}_{f}=\boldsymbol{G}_{f}^{+} Y
$$

where $\boldsymbol{G}_{f}^{+}$is the pseudo inverse matrix of $\boldsymbol{G}_{f}$. Once $\overline{\boldsymbol{S}}_{f}$ is determined, the desired estimation result $\boldsymbol{s}(n-k)$ can be obtained from $S_{f}(n)$ by

$$
\widehat{\boldsymbol{S}}_{f}(n)=q_{\Phi}\left\{\boldsymbol{U} \hat{\overline{\boldsymbol{S}}}_{f}(n)\right\}
$$

Finally, we implement the decision on $\widehat{\boldsymbol{S}}_{f}$, which means that the part ISI is eliminated and the results are more reliable.

\section{Joint iterative separation and decoding algorithm}

The prior information of symbol is helpful to improve the separation efficiency. Fig. 2 is a block diagram of joint iterative separation and decoding, and iteration is performed by constantly interacting soft information between the blind separation and decoding modules. In order to reduce the computational complexity, we use the separation algorithm based on lattice reduction at the first time and then the separated symbols are fed into the decoding module. In the latter iteration, the separation is performed using the PSP algorithm. The blind separation module is processed according to the received mixed signal $\mathbf{r}$ and a priori information of symbol $L_{s}^{a}\left(s_{i, k}\right)$, and calculates bit information $L_{d}^{a}\left(c_{i, k J+j}\right)$ that is output to the decoding module. The decoding module calculates the posterior information $L_{d}\left(c_{i, k J+j}\right)$ of each bit according to the decoding algorithm, and then subtracts the prior information $L_{d}^{a}\left(c_{i, k J+j}\right)$ to obtain the external information $L_{d}^{e}\left(c_{i, k J+j}\right)$. After mapping, a priori information $L_{s}^{a}\left(s_{i, k}\right)$ is formed, which is fed back to the blind separation module and the next iteration is started.

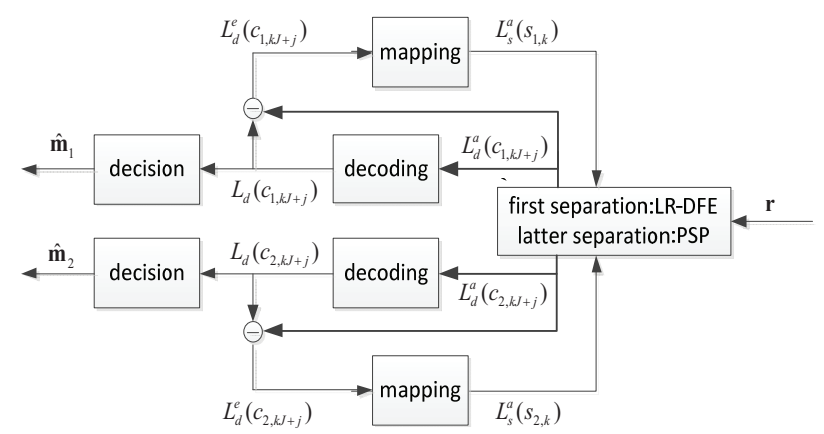

Fig. 2 block diagram of joint iterative separation and decoding

In the above block diagram, blind separation and decoding are two key modules. The decoding module can adopt the existing mature algorithm, and the blind separation module will be described below.

In order to iteration, the separation here requires the soft input values and provides soft output. Using the bit likelihood metric, the bit information of the output can be represented as

$$
L_{d}^{a}\left(c_{i, k J+j}\right)=\ln \frac{p\left(c_{i, k J+j}=1 \mid r_{0: 2 K-1}\right)}{p\left(c_{i, k J+j}=0 \mid r_{0: 2 K-1}\right)}
$$

where $K$ is the total number of symbols for the data segment to be processed. We take $r_{2 k: 2 k+3}$ four points, then the formula (10) can be rewrite to

$$
L_{d}^{a}\left(c_{i, k J+j}\right)=\ln \frac{p\left(c_{i, k J+j}=1 \mid r_{2 k: 2 k+3}\right)}{p\left(c_{i, k J+j}=0 \mid r_{2 k: 2 k+3}\right)}
$$

We can futher write the formula(10) into 


$$
\begin{aligned}
& L_{d}^{a}\left(c_{i, k J+j}\right)=\ln \frac{p\left(c_{i, k J+j}=1 \mid r_{2 k: 2 k+3}\right)}{p\left(c_{i, k J+j}=0 \mid r_{2 k: 2 k+3}\right)} \\
& =\ln \frac{\sum_{S_{k}: c_{i, k J+j}=1} p\left(S_{k} \mid r_{2 k: 2 k+3}\right)}{\sum_{S_{k}: c_{i, k J+j}=0} p\left(S_{k} \mid r_{2 k: 2 k+3}\right)}
\end{aligned}
$$

where $S_{k}$ is the sequence of symbols corresponding to $r_{2 k: 2 k+3}$, then we can get

$$
\begin{aligned}
& p\left(S_{k} \mid r_{2 k: 2 k+3}\right) \propto p\left(r_{2 k: 2 k+3} / S_{k}\right) \cdot p\left(S_{k}\right) \\
& =\prod_{l=2 k}^{2 k+3} p\left(r_{l} / S_{k}\right) \cdot \prod_{l=k+1-L_{1}}^{k+L_{2}+1} p\left(s_{1, l}\right) p\left(s_{2, l}\right) \\
& =\prod_{l=2 k}^{2 k+3} \frac{1}{\sqrt{\pi} \sigma} \exp \left(-\frac{\left|r_{l}-\hat{r}_{l}\right|^{2}}{\sigma^{2}}\right) \cdot \prod_{l=k+1-L_{1}}^{k+L_{2}+1} \prod_{j=0}^{J-1} p\left(c_{1, l J+j}\right) p\left(c_{2, l J+j}\right)
\end{aligned}
$$

where $\sigma^{2}$ is the noise variance, and $\hat{r}_{l}$ is the reconstructed value of $r_{l}$, which is calculated as following according to the formula(6)

$$
\hat{r}_{2 k+m}=\sum_{n=1-L_{1}}^{L_{2}} s_{1, k+n} \hat{f}_{1,2 k+m}(n)+\sum_{n=1-L_{1}}^{L_{2}} s_{2, k+n} \hat{f}_{2,2 k+m}(n)
$$

where $S_{i, k+n}$ is derived from sequence $S_{k}$, and $\hat{f}_{i, 2 k+m}(n)$ is the estimate of $f_{i, 2 k+m}(n)$, which can be tracked using the LMS algorithm.

Bring the formula(13) into the formula(12) and use the approximate formula

$$
\ln \left(e^{x}+e^{y}\right) \approx \max (x, y)
$$

Then we can get

$$
\begin{aligned}
& L_{d}^{a}\left(c_{i, 2 k+j}\right) \approx \max _{S_{k}: c_{i, 2 k+j}=1}\left(-\frac{1}{\sigma^{2}} \sum_{l=2 k}^{2 k+3}\left|r_{l}-\hat{r}_{l}\right|^{2}\right. \\
& \left.+\sum_{l=k+1-L_{1}}^{k+L_{2}+1} \sum_{j=0}^{J-1}\left(c_{1, l J+j} L_{d}^{e}\left(c_{1, l J+j}\right)+c_{2, l J+j} L_{d}^{e}\left(c_{2, l J+j}\right)\right)\right) \\
& -\max _{S_{k}: c_{i, 2 k+j}=0}\left(-\frac{1}{\sigma^{2}} \sum_{l=2 k}^{2 k+3}\left|r_{l}-\hat{r}_{l}\right|^{2}+\right. \\
& \left.\sum_{l=k+1-L_{2}+1}^{k+1} \sum_{j=0}^{J-1}\left(c_{1, l J+j} L_{d}^{e}\left(c_{1, l J+j}\right)+c_{2, l J+j} L_{d}^{e}\left(c_{2, l J+j}\right)\right)\right)
\end{aligned}
$$

Obviously, limiting the range of the $S_{i}$ can reduce the amount of calculation with some performance loss. In the first separation, we use decision feedback algorithm based on the lattice reduction, which is achieved by fixing the symbol of the preceding time. In the latter separation, we can fix the symbol before and after target symbol because the entire sequence of symbols has been estimated. The amount of calculation in blind separation brought from iterative process can be negligible, thus greatly improve the detection performance.

\section{Simulation results}

This section presents the performance of the algorithm which is evaluated through simulation using two QPSK modulated signals. We first compare the performance of the two methods of separation. We suppose that LDPC encoding is adopted.

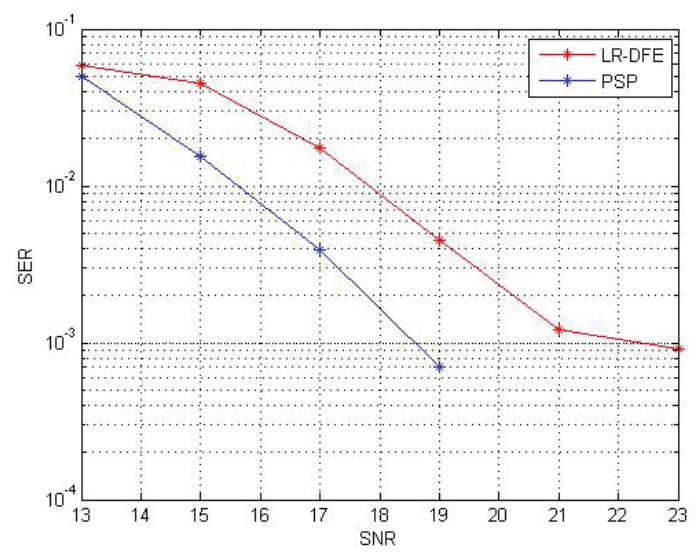

Fig. 3. The separation performance of LR-DFE algorithm comparing with PSP

As can be seen from Figure 3, when the bit error rate is $10^{-3}$, LR-DFE has a $2.2 \mathrm{db}$ performance penalty relative to the PSP algorithm, but the computation is reduced by 10 times[8], so it can be used to process higher order modulation signals, such as 8QAM, 16QAM, so the LRDFE algorithm has better engineering applicability.

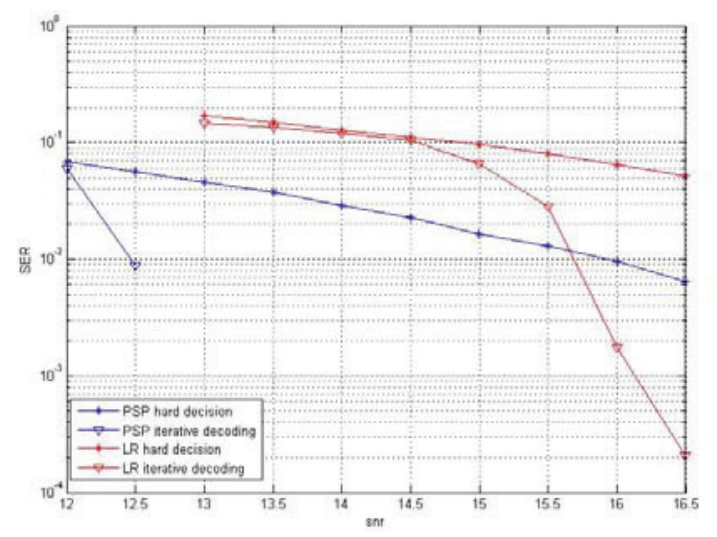

Fig. 4. The performance of joint iterative separation and decoding algorithm

Last, the performance of joint iterative separation and decoding algorithm is investigated. As mentioned earlier, in the first separation, we fix the symbol before the target symbol and in the later separation, we fix the symbol before and after the target symbol. In the first separation, we use different separation methods, one is LR-DFE, and the other is PSP. As can be seen from the diagram, the iterative decoding brings huge improvement to the performance of separation, and the maximum bit error rate can be reduced from $9.62 \mathrm{E}-2$ to $6.55 \mathrm{E}-2$ at an iteration, and achieve generally error free at two iteration.

\section{Conclusion}

A low complexity joint iterative and decoding algorithm is proposed in this paper, It's complexity is 10 times lower than that of PSP iterative decoding, so it has good practicability. Simulation results show that under the same number of iterations, the performance loss is $2.2 \mathrm{db}$ 
Compared with PSP. However, iterative decoding can bring 1.5-1.2db gain, so the effectiveness of the algorithm is proved.

\section{References}

1. Mark D. Paired carrier multiple access(PCMA) for satellite communication[C]. Pacific Telecommunications Conference, Honolulu, Hawaii, 1998: 787-791.

2. H.Yao,G.W.Wornell.Lattice-reduction-aided detectors for MIMO communication systems[C].IEEE Global Telecommunication Conference, Taipei,2002,424-428.

3. C.Windpassinger,R.F.H.Fischer.Low-complexity near-maximum-likelihood detection and precoding for MIMO systems using lattice reducation[C].IEEE Information Theory Workshop(ITW),Paris, 2003,345-348.

4. D. Wubben,R.Bohnke,V.Kuhn,et al..NearMaximum-Likelihood Detection of MIMO Systems Using MMSE-Based Lattice Reduction[C].IEEE International Conference on Communication(ICC),Paris,2004,798-802.

5. Qin X B, He G H, Ma J. The application of Lattice Reduction Algorithm in MIMO detection[J]. Chinese scientific papers, Vol.10, No.14, 2015.

6. Liao C H, Tu S L, Wan J. Single channel blind separation/decoding Algorithm based on iterative mixed frequency signals[J]. Journal of communications, 2011, 32(8): 111-117.

7. Zhao Y F, Cao Y J, Dai X C. Single Antenna Interference Cancellation Algorithm Based on Lattice Reduction[J]. Journal of Communications, 2015:93-100.

8. C. P. Schnorr and M. Euchner, "Lattice basis reduction:Improved practical algorithms and solving subset sum problems,"Mathematical programming, vol. 66, no. 3, pp. 181-199, 1994. 